\title{
Efectos del Programa Creciendo Fuertes sobre el bienestar psicológico y autoeficacia generalizada en adolescentes: estudio preliminar
}

\section{Effects of the Programa Creciendo Fuertes on psychological well-being and generalized self-efficacy in adolescents: preliminary study}

\author{
Diego García-Álvarez \\ $\triangle$ diegogarcia_123@hotmail.com \\ Centro de Investigación y Experimentación Pedagógica (CIEP), Uruguay. \\ María José Soler id \\ Asociación Jóvenes Fuertes, Montevideo, Uruguay \\ Rubia Cobo-Rendón (iD) \\ Universidad de Concepción, Programa de Doctorado en Psicología, \\ Programa de Doctorado en Psicología, Chile.
}

Recibido: 03-04-2018

Aceptado: 06-06-2018

Publicado: 08-06-2018

\section{RESUMEN}

Objetivo. El propósito de este artículo es dar a conocer el diseño, ejecución y determinación de los efectos preliminares de un programa basado en las fortalezas del carácter. Los objetivos de esta investigación son: a) caracterizar los niveles de bienestar psicológico y autoeficacia generalizada antes y después de la aplicación del Programa Creciendo Fuertes, b) determinar el efecto dicho programa sobre el bienestar psicológico y autoeficacia generalizada de adolescentes de secundaria y c) analizar la relación de la virtud del coraje con el bienestar psicológico y la autoeficacia generalizada luego de realizado el programa en los estudiantes participantes. Metodo. Metodológicamente, se ajusta a un diseño de investigación cuasi experimental de estudio pre - post con un solo grupo, compuesto por una muestra de veinticuatro adolescentes estudiantes de secundaria. Se emplearon instrumentos de recolección de datos con adecuada confiabilidad y validez. Resultados. Se presentaron incrementos estadísticamente significativos en los niveles de las variables de bienestar psicológico y autoeficacia generalizada luego de aplicar el programa. También se mostraron diferencias estadísticamente significativas en las puntuaciones de estas variables antes y después de aplicar el programa. A su vez, se presentaron correlaciones estadísticamente significativas entre la virtud del coraje, autoeficacia generalizada y bienestar psicológico. Conclusiones. De acuerdo con estos resultados preliminares, se concluye que el programa puede ser empleado a modo de refuerzo psicoeducativo para la promoción del bienestar en las escuelas secundarias.

Palabras clave: bienestar del estudiante, autoeficacia, programas educativos, educación del carácter. 
García-Álvarez et al - Efectos del programa Creciendo Fuertes sobre el bienestar psicológico y autoeficacia

\section{ABSTRACT}

Objetive. The purpose of this article is to present the design, execution and estimate of the preliminary effects of the program based on the strengths of character. The objectives of this research are: a) characterize the levels of psychological well-being and generalized self-efficacy before and after the application of the Programa Creciendo Fuertes (Growing Strong Program), b) determine the effectiveness of the program on the psychological well-being and generalized self-efficacy of high school adolescents, and c) analyze the relationship of the virtue of courage with psychological well-being and generalized self-efficacy after carrying out the program in the participating students. Method. Methodologically, it is adjusted to a quasi - experimental research design of a pre - post study with a single group with a sample of 24 secondary school adolescents. Data collection instruments were used with adequate reliability and validity. Result. By way of results, there are statistically significant increases in the levels of the variables of psychological well-being and generalized self-efficacy in comparison between the pre- and post-program measures. There were also statistically significant correlations between the virtue of courage, generalized self-efficacy and psychological well-being. Conclusions. According to these preliminary results, that the program can be used as a psycho-educational effort to promote well-being in secondary schools.

Keywords: student welfare, self-efficacy, educational programs, character education.

\section{INTRODUCCIÓN}

La Psicología Positiva surge como un enfoque científico centrado en el estudio sistemático, serio y riguroso de los aspectos positivos, rasgos de la personalidad, del bienestar (Seligman, 2011) y de la experiencia óptima (Csikszentmihalyi, 1997). En un primer momento, los pilares de la Psicología Positiva fueron las tres vidas: placentera, comprometida y con sentido, con un eje transversal denominado "las virtudes y fortalezas del carácter"; luego este modelo teórico de la auténtica felicidad se reformuló para dar paso a la teoría PERMA (Seligman, 2002; 2011), por sus siglas en inglés, a saber: emociones positivas, compromiso, relaciones interpersonales, sentido y logros. En esta teoría se considera el bienestar como un constructo multidimensional y relacional, y se mantiene el eje transversal de las virtudes y fortalezas del carácter, entendidas como rasgos de la personalidad con valor moral que se vinculan naturalmente con el bienestar psicológico, (Peterson y Seligman, 2004).

Por su parte, Fernández Ríos (2008, p. 65) presenta un concepto de Psicología Positiva con él cual merece la pena comenzar este trabajo de investigación: "La Psicología Positiva es el estudio científico de los mecanismos psicológicos de las fuerzas, recursos o virtudes que, en un contexto sociomaterial, contribuyen a construir un funcionamiento óptimo de individuos, grupos, organizaciones y sociedades". De ahí que este autor exponga que la Psicología Positiva viene a promover un cambio en las principales escuelas de la psicología que habían estado centradas en el estudio de la enfermedad, la patología y situaciones cimentadas en emociones negativas desde el abordaje de los modelos de déficit o de vulnerabilidad del ser humano, para dar paso a una psicología más orientada a modelos humanistas, constructivos y esperanzadores del ser humano, más encuadrada en la prevención que a la rehabilitación. 
Desde esa perspectiva, Palomera (2017) expone que uno de los pilares de la Psicología Positiva está compuesto por las instituciones educativas, pues es allí en donde los estudiantes transcurren la mayor parte de su tiempo y deberían tener la oportunidad de aprender habilidades para la vida, para adaptarse a la sociedad e incluso aprender a ser felices. De igual manera, Cobo y García (2017) explican que los postulados de la teoría PERMA del bienestar propuesta por Seligman (2011) pueden ser operacionalizados en técnicas, dinámicas y estrategias a ser llevadas a cabo por los docentes en su praxis didáctica diarias, con énfasis en una actitud positiva por parte de los docentes para visualizar sus aulas de clases como espacios para el cultivo de las fortalezas del carácter en miras de la promoción del bienestar -felicidad- de sus estudiantes. A esto agregan Moreno y Gálvez (2010, p. 212): "la felicidad también se aprende".

Por consiguiente, se han desarrollado a nivel mundial distintas experiencias fundamentadas en la Psicología Positiva aplicada a la educación, ya sea a modo de programas para hacer trabajados en los mismos horarios de clases o programas con una carga horaria distinta. Entre los programas -con adecuada percepción acerca de sus efectos- en la literatura científica especializada se pueden nombrar: Penn Resiliency Program y el Strath Haven Positive Psychology Curriculum realizados por Seligman et al. (2009), basados en la promoción de las fortalezas del carácter y la resiliencia en niños y adolescentes con efectos demostrados en el aumento del bienestar; el programa Aulas Felices de España, creado por Arguís, Bolsas, Hernández y Salvador (2011); en Estados Unidos de América, el programa Smart Strengths (Yeager, Fisher y Shearon, 2011); en Venezuela, el programa de Promoción Positiva de la Adolescencia: Adolescentes Cuentan (Universidad Metropolitana y Fondo de Naciones Unidas para la Infancia, 2012), y en Australia, las experiencias de los programas que describe Palomera (2017) en su revisión: Geelong Grammar School, proyecto pionero en ese país basado en la educación del bienestar, y Bounce Back, dirigido a promover el bienestar y la resiliencia desde la educación inicial hasta la secundaria.

Ahora bien, la mayoría de las intervenciones diseñadas han sido destinadas a la educación secundaria, de manera que los adolescentes conforman la población usuaria de los mismos. De igual manera, el Programa Creciendo Fuertes que se presenta en este trabajo de investigación está diseñado para esta etapa del ciclo vital. La adolescencia es un periodo crítico del desarrollo que puede incluir ciertos factores de riesgo psicosocial que pueden conllevar depresión, ansiedad, consumo de sustancias ilícitas, relaciones sexuales sin protección, violencia y otras situaciones asociada a conductas de riesgo (Papalia y Feldman, 2012; Organización Mundial de la Salud, 2011).

Sin embargo, la literatura también sugiere que los adolescentes presentan fortalezas de la personalidad que pueden actuar como elementos positivos-protectores en procesos de resiliencia ante eventos adversos durante esta etapa del ciclo vital (Quiceno y Vinaccia, 2013; Rivers y Cowie, 2006; López, Pérez y D’Aubeterre, 2010; López, 2012). En efecto, el propósito de esta intervención es promover esos factores de protección. De acuerdo con Papalia, Feldman y Martorell (2012), la adolescencia es una etapa del ciclo vital con oportunidades de crecimiento, asociadas a factores de protección; pero a su vez con peligros psicosociales, relacionados a factores y conductas de riesgo que pueden afectar el bienestar físico y psicológico. 
En ese orden ideas, la adolescencia se ubica en un contexto sociocultural que actualmente se caracteriza por el incremento de factores de riesgos y conductas riesgosas, a saber: sexo sin protección, conductas inadecuadas en alimentación, consumo de cigarrillos y alcohol, así como consumo de sustancias ilícitas, crisis de valores, embarazos no deseados, fracaso escolar y excesivo hedonismo, entre otros factores (Vargas y GonzálezTorres, 2009), razón por la cual se ha reflexionado sobre la pertinencia de la educación del carácter como una estrategia que busque preparar a los estudiantes para la vida.

En relación al concepto de educación del carácter, este se ha concebido desde enfoques generales que van evolucionando la educación en valores, ciudadanía, educación emocional y afectiva (Vargas y González-Torres, 2009) y últimamente a los enfoques de las habilidades para la vida, y educación socio-emocional y para el bienestar (Bisquerra Alzina, 2010; Muñoz de Morales y Bisquerra, 2013; García y Serrano, 2017). De modo que existe consenso sobre la característica de que la educación del carácter está vinculada a la formación del buen carácter, de la noción de hábito-virtud y de una educación que permita a los educandos diferenciar sobre la adecuación de hábitos con relación a la salud. Desde el surgimiento de la Psicología Positiva, como un enfoque centrado en el estudio científico del bienestar, se ha revitalizado el concepto de educación del carácter (Bernal, González-Torres y Naval, 2015; Peñacoba-Arribas y Santa-Cruz Vera, 2016), puesto que la Psicología Positiva busca la promoción del bienestar a través del cultivo de las fortalezas del carácter. A este punto, cabe destacar que existe una sólida base empírica que demuestra la relación de las fortalezas del carácter con el bienestar psicológico (Giménez, 2010; Toner, Haslam, Robinson, y Williams, 2012).

En este trabajo de investigación se asume una educación del carácter, emocional y para el bienestar como estrategia de prevención inespecífica que puede tener efectos beneficiosos frente al reto de la prevención múltiple ante los variados factores y conductas de riesgos a la que actualmente la adolescencia está expuesta (García y Serrano, 2017; Cobo y García, 2017; Bisquerra Alzina y Hernández Paniello, 2017; Bisquerra, 2010; Seligman, Ernst, Gillham, Reivich, y Linkins, 2009; Ruiz-Aranda, Cabello, Salguero, Castillo, Extremera y Fernández-Berrocal, 2010). Además, Liccioni y Soto (2006, p. 28) definen la educación emocional como una "[...] forma de prevención primaria inespecífica consistente en intentar minimizar la vulnerabilidad a las disfunciones o prevenir su ocurrencia"; es decir que la meta principal es educar a las personas en comportamientos preventivos en pro del desarrollo humano.

De lo anterior se desprende que este estudio se justifica dado que la investigación en Psicología Positiva, como disciplina aplicada a la psicología educacional, debe ir orientada a elaborar modelos teóricos y profundizar en las dimensiones teórico-práctico-técnicas para generar conocimientos para ser considerados en el diseño de estrategias y programas de intervención en pro de la mejora de las prácticas educativas (Díaz Barriga, 2009). A su vez, los resultados obtenidos buscan aportar a otros estudios y experiencias científicas en las líneas de investigación sobre promoción del bienestar psicológico en ámbitos educativos formales en concordancia con los antecedentes uruguayos de García y Soler (2016).

Por lo antes expuesto, las preguntas de investigación que guían este trabajo son: a) ¿Cuál será el nivel de bienestar psicológico y autoeficacia generalizada antes y después de la aplicación del Programa Creciendo Fuertes? b) ¿Cuál será el efecto del Programa Creciendo Fuertes en el bienestar psicológico y autoeficacia generalizada de 
los adolescentes de secundaria? Y por último, ¿cuál será la relación de la virtud del coraje con el bienestar psicológico y la autoeficacia generalizada en los estudiantes adolescentes luego de la aplicación del mismo?

Por su parte, los objetivos de esta investigación son: a) caracterizar los niveles de bienestar psicológico y autoeficacia generalizada antes y después de la aplicación del Programa Creciendo Fuertes, b) determinar el efecto del Programa Creciendo Fuertes sobre el bienestar psicológico y autoeficacia generalizada de adolescentes de secundaria y c) analizar la relación de la virtud del coraje con el bienestar psicológico y la autoeficacia generalizada luego de realizado el Programa Creciendo Fuertes en los estudiantes participantes.

Por consiguiente, el sistema de hipótesis estuvo conformado de la siguiente manera: a) los participantes registrarán mayores niveles de bienestar psicológico y autoeficacia generalizada luego de la aplicación del Programa Creciendo Fuertes en comparación con las medidas de pre aplicación. b) Las relaciones serán significativas entre la virtud del coraje y las variables bienestar psicológico y autoeficacia generalizada luego de la aplicación del Programa Creciendo Fuertes.

\section{Psicología positiva: bienestar psicológico y autoeficacia}

Desde años recientes la psicología ha mostrado un amplio interés por la investigación sobre aspectos positivos propios de la experiencia humana, los cuales permiten a las personas y sociedades desarrollarse y crecer (Contreras y Esguerra, 2006). La Psicología Positiva como disciplina científica ha desarrollado investigación en diversos ámbitos como el educativo, organizacional y de salud. Entre sus variables de mayor interés se encuentra el estudio del bienestar en estos contextos. El bienestar puede ser visto como las evaluaciones subjetivas que las personas realizan sobre la vida que llevan (McMahan y Estes, 2011).

Históricamente la autoeficacia ha sido el principal elemento de la tesis del determinismo recíproco, propuesto por Albert Bandura, definido como el juicio o las creencias que un individuo tiene sobre sus propias capacidades, con base a las cuales este movilizará su energía, organizará y ejecutará sus actos, aprovechando los recursos cognitivos con el curso de acción necesaria, de modo que le permitan alcanzar el objetivo deseado (Bandura, 1997). Los factores personales y conductuales, al igual que los ambientales producen una interrelación e interdependencia durante este proceso y conforman una triada de determinación recíproca.

Existen cuatro fuentes de autoeficacia: (1) las experiencias de dominio, basadas en el recuerdo de la superación de obstáculos a través del esfuerzo perseverante; (2) el modelado social (ver a la gente tener éxitos similares a uno mismo por el esfuerzo perseverante); (3) la persuasión social (si la gente se convence en creer en sí mismo, es más perseverante en medio de las dificultades, lo que aumenta las posibilidades de éxito), y (4) la evaluación de los estados físicos y emocionales, en la que las creencias de autoeficacia se fortalecen mediante la reducción de la ansiedad y la depresión (Bandura, 2012). La autoeficacia es fundamental para fomentar en el individuo el sentimiento que tiene de libertad de acción (agencia) para influir en su vida (Alegre, 2013). 
En este caso las personas son entendidas como productores y productos de sus ambientes y de sistemas sociales, son agentes de cambio en sus vidas y en sus entornos a partir de la proactividad, la autorreflexión y la autorregulación, en lugar de ser organismos conducidos por instintos internos escondidos o modulados por fuerzas ambientales (Ornelas Contreras, Blanco Venga, Peinado Pérez y Blanco Ornelas, 2012). Estos argumentos concuerdan con algunas evidencias en el estudio del bienestar en que se describe que el $40 \%$ de la varianza en la frecuencia de la actividad intencional permiten ofrecer mayores oportunidades de experimentar altos niveles de este (Lyubomirsky y Sheldon, 2005), es decir que las actividades intencionales que realizan las personas tienen un impacto importante en la presencia del bienestar en sus vidas. Asimismo, Fernández Ríos (2008) plantea que la Psicología Positiva concibe al ser humano como agente responsable de cambio.

Significa entonces que el bienestar puede ser incrementado por tres grandes factores: la genética, las circunstancias de la vida y las actividades deliberadas. Estas últimas ofrecen una ruta posible para el incremento y mantenimiento del bienestar en la vida de las personas (Lyubomirsky y Sheldon, 2005). De esta forma, la asociación entre bienestar y los aportes de la teoría social cognitiva, en especial de la autoeficacia, estaría dimensionada a las conductas deliberadas que realiza la persona dentro de su contexto, otorgándole la capacidad de influir en su entorno, así como este influye en la persona; es decir que el bienestar humano y los logros son productos de una reciprocidad intrapersonal, conductual y ambiental (Bandura, 2011).

La autoeficacia es un concepto significativo en cuanto al control de las demandas académicas, por lo tanto, es un elemento a considerar en el que influyen procesos psicológicos, cognitivos, motivacionales y afectivos que juegan un papel importante tanto en la toma de decisiones, como en el manejo del estrés, en las actividades académicas cotidianas y en muchos otros factores que intervienen sobre la forma en que se perciben y procesan las experiencias.

Estudios confirman la relación del bienestar con las creencias de autoeficacia, correspondencia que se ha revelado como un factor clave en el mantenimiento del bienestar en la vida, este constructo reduce las emociones negativas y aumenta las positivas, las cuales incrementan la competencia percibida, la cual disminuye a su vez los estados de ánimo negativos (Romero, Cruz, Gallardo, y Peñacoba, 2013; González Cabanach, Valle Arias, Freire Rodríguez, y Ferradás Canedo, 2012). Las creencias de eficacia también afectan lo que se piensa sobre uno mismo y cómo se siente con estos pensamientos. En este sentido, la autoeficacia sería una de las características personales que promueven el bienestar de las personas, aun en condiciones de adversidad (Chavarría y Barra, 2014).

\section{MÉTODO}

Diseño. El diseño de esta investigación se enmarca en una metodología cuasi experimental de estudio pre-post con un solo grupo (Montero y León, 2002; León y Montero, 2015) debido a la finalidad de conocer el efecto del Programa Creciendo Fuertes sobre las variables de bienestar psicológico y autoeficacia generalizada, entonces se tomó una 
medida de ambas antes (pre) de la aplicación del programa basado en las fortalezas del carácter y otra medición luego de finalizar (post). Vale la pena destacar que la investigación se considera también de tipo correlacional pues se buscó identificar la fuerza, magnitud y significancia entre las fortalezas de la virtud del coraje contenida en el Programa Creciendo Fuertes y las variables de bienestar psicológico y autoeficacia, (Hernández, Fernández y Baptista, 2008).

Participantes. La muestra estuvo conformada por un grupo de veinticuatro estudiantes adolescentes de primer año de secundaria, todos del sexo masculino, la media de edad fue de 12,5 años, específicamente las edades fueron: doce años (60\%), trece años $(20 \%)$ y catorce años $(10 \%)$.

Instrumentos. Se empleó una encuesta sociodemográfica compuesta por escalas, a saber: escala bienestar PERMA para adolescentes, de Soler (2016), basada en la teoría del bienestar de Seligman (2011) que mide el bienestar psicológico en las dimensiones de emociones positivas, flujo, relaciones interpersonales, sentido de vida y metas, cada sub escala con adecuados índices de consistencia interna por el alfa de Cronbach, así como la escala total. De igual forma, se incluyó una escala que evalúa la virtud del coraje en adolescentes de Soler (2016) en sus dimensiones de perseverancia, honestidad y valentía basada en la teoría de las virtudes y fortalezas del carácter de Peterson y Seligman (2004), también cuenta con adecuados índices de consistencia interna en el alfa de Cronbach. Además, se evaluó la autoeficacia generalizada en adolescentes a través de la escala de Baessler y Schwarzer (1996) de validación española de Oliva et al. (2011), con adecuados índices de confiabilidad y validez.

Procedimiento. Se inició el proceso con las gestiones técnicas, administrativas y éticas con relación a los permisos necesarios en la institución educativa (brindar información del proyecto, cumplir con los procedimientos relacionados a consentimiento informado a nivel institucional, con representantes legales y con cada estudiante, y otros requisitos éticos como la participación voluntaria, no causar molestias, el aseguramiento de anonimato y confidencialidad, y la explicación de consentimiento informado válido). Posteriormente se comenzó con la recolección de datos mediante la colaboración de los talleristas que aplicaron los instrumentos de recolección al comienzo y al final del Programa Creciendo Fuertes (abril-septiembre). Por otro lado, los autores anuncian que no existe conflicto de interés.

Análisis de datos. Los datos fueron trabajados en el programa estadístico IBM SPSS Statistics 23.0. Se desarrollaron estadísticas descriptivas y pruebas de bondad de ajuste para determinar la existencia de distribución normal para tomar la decisión de aplicar análisis paramétricos o no paramétricos, es decir, el análisis de correlaciones para conocer las relaciones entre las variables y la prueba de contraste de hipótesis, para conocer el efecto del programa sobre las variables "bienestar psicológico" y "autoeficacia", tomando como referencias las orientaciones de León y Montero (2015) y Hernández, Fernández y Batista (2008) para el cumplimento de los objetivos de acuerdo al diseño planteado.

Programa Creciendo Fuertes. El Programa Creciendo Fuertes está basado en los fundamentos teóricos de las virtudes y fortalezas del carácter de Peterson y Seligman (2004). Inicialmente el programa fue pensado, diseñado y planificado para estudiantes adolescentes del ciclo básico de secundaria (primero a tercer año, nivel educativo 
obligatorio para adolescentes entre doce y catorce años edad en Uruguay). El propósito del programa es viabilizar la formación de un carácter sano, mediante la identificación, reconocimiento y puesta en práctica cotidiana de las fortalezas del carácter en los estudiantes.

A nivel operativo, el Programa Creciendo Fuertes contiene las veinticuatro fortalezas de carácter que se encuentran organizadas en tres años académicos, y toma en cuenta el desarrollo evolutivo e intereses de los usuarios (véase García, Soler y Achard, 2017). De este modo, en cada año académico se promocionan de siete a ocho fortalezas, cubriendo las veinticuatro a lo largo del ciclo básico de la educación secundaria. De ahí que el programa sea diseñado bajo intervenciones en modalidades de talleres vivenciales facilitados por dos talleristas fijos (formados previamente e incluso con guías estandarizadas de protocolo), que trabajan con el mismo grupo a lo largo del año. La duración de cada taller es de un módulo -dos horas clase- (ochenta minutos aproximadamente). Previo al comienzo del programa de talleres Creciendo Fuertes, se les aplica a todos los estudiantes el cuestionario VIA dirigido a jóvenes de la Universidad de Pennsylvania para que cada uno descubra sus cinco fortalezas preponderantes.

En este trabajo, solo se va a presentar el Programa Creciendo Fuertes diseñado para primer año de ciclo básico de acuerdo a los objetivos de investigación planteados. En ese sentido, se comienza el año con un primer taller denominado "Me conozco", de carácter introductorio (acá para efectos de investigación se hizo la primera medición); este tiene como objetivo reflexionar acerca de las distintas dimensiones del ser humano y la responsabilidad personal que cada uno tiene en dirigir su propia vida hacia el desarrollo pleno. A partir de ahí, comienzan los talleres relacionados al conjunto de fortalezas seleccionadas para cada nivel.

La organización del Programa Creciendo Fuertes correspondiente al primer año se realiza a modo de sesiones, cada una dedicada exclusivamente a una fortaleza, con la siguiente secuencia:

0. Taller de autoconocimiento, introductorio (acá se hizo primera medición considerada pre)

1. Valentía.

2. Perseverancia.

3. Autenticidad.

4. Gratitud.

5. Autocontrol.

6. Inteligencia social.

7. Humor (acá se realizó la segunda medición considerada post).

Para efectos de investigación se consideró la virtud del coraje en sus fortalezas: valentía, perseverancia y autenticidad, como variable para ser medida y correlacionada con las variables de bienestar psicológico y autoeficacia generalizada.

El Programa Creciendo Fuertes está dirigido a los estudiantes de secundaria, pero con la intención de que los docentes tengan la posibilidad de trabajar de manera transversal las fortalezas del carácter de acuerdo a los contenidos a enseñar desde cada una de sus asignaturas, estos reciben el taller con la fortaleza del carácter que a su vez están trabajando los estudiantes, e incluso se les hace entrega de unos cuadernillos 
llamados "Guías del educador" que reseñan teóricamente la fortaleza del mes y formas de promoverla en el salón de clases. De modo que por mes se tienen: una instancia de trabajo con los docentes, una instancia de trabajo con los estudiantes con la fortaleza correspondiente y luego otra sesión de apoyo con los estudiantes para consolidar la fortaleza.

Para entrar en detalle, por cada taller se define la fortaleza para que los estudiantes tengan una comprensión concreta y pragmática. Esto se hace por medio de dinámicas y juegos para que los adolescentes construyan su propio aprendizaje. Una vez que los estudiantes aprehenden lo que implica la fortaleza, se reflexiona sobre acciones concretas a través de las cuales se concretizaría la misma. Asimismo, se reflexiona sobre los beneficios y consecuencias al incorporar la fortaleza en el comportamiento cotidiano; este conocimiento hace que la integración de la fortaleza sea algo que quiera ser alcanzado o logrado y por tanto se transforma en un objetivo para los jóvenes (valor intrínseco). Los objetivos se hacen explícitos y conscientes durante cada taller, dando lugar a propósitos voluntarios por parte de los estudiantes que son anotados en un cuaderno, para luego ser operacionalizados en acciones concretas para llevar a cabo durante la semana.

Siguiendo con esa idea, el propósito final de estos talleres es la práctica de acciones por parte de los estudiantes, que eventualmente serán transformadas en hábitos operativos adecuados para su desarrollo e integrados a su carácter. Luego, en la sesión de apoyo con los mismos talleristas se analiza cómo transcurrió la semana con respecto a los propósitos planteados y se profundiza acerca de la fortaleza. Se continúa trabajando durante el resto del mes sobre la misma.

Al iniciar cada taller se parte de los conocimientos previos que los estudiantes tienen acerca de la fortaleza. Desde ese lugar y a través de distintas dinámicas se van realizando esfuerzos didácticos para que los estudiantes construyan su propio conocimiento de la fortaleza. Otro punto a destacar es que se busca implicar a los estudiantes en el tema, utilizando ejemplos que tengan relación con sus intereses y preocupaciones y que de alguna manera puedan proyectar en alguna situación personal en la que entre en juego la fortaleza en cuestión. También se busca el aprendizaje contextualizado para que tengan una verdadera aprehensión de la fortaleza. Las actividades propuestas tienen como objetivo didáctico relacionar lo que aprehendieron con sus experiencias en la vida diaria para lograr así un aprendizaje significativo.

En cuanto a estrategias organizativas, en el Programa Creciendo Fuertes se utilizan distintas formas de agrupamiento. En muchas instancias se recurre al trabajo en pequeños grupos debido a que los mismos ofrecen múltiples ventajas además de oportunidades para comenzar a poner en práctica las fortalezas. Una gran parte de las dinámicas planteadas requiere también del trabajo en contextos de colaboración. Algunas veces el trabajo implica una reflexión individual; en estas instancias se les da a los estudiantes la posibilidad de elegir si quieren compartir o no sus reflexiones, debido a que muchas veces se tratan temas que pueden llegar a ser movilizadores para algunos.

En cuanto a los lugares utilizados para los talleres, se trabaja tanto en salones de clase como en salones multiuso. Estos últimos propician el trabajo en la modalidad de taller permitiendo el movimiento del mobiliario. Es necesario para los talleres contar con una 
García-Álvarez et al - Efectos del programa Creciendo Fuertes sobre el bienestar psicológico y autoeficacia

pantalla dispuesta al frente del salón y, en el caso que no se disponga de la misma, los talleristas se encargan de llevar el material necesario. Los talleres son acompañados de una presentación tipo Power Point teniendo en cuenta las edades de los participantes y, por tanto, el tipo de recursos a utilizar para favorecer la atención.

Al finalizar cada taller se entrega a los estudiantes una hoja de evaluación en la cual se recoge la opinión acerca del taller, recursos, actitudes de los talleristas, materiales y otros. El objetivo de la evaluación es en primer lugar detectar algún cambio o ajuste que se deba hacer al taller de acuerdo al grupo, así como también conocer más a los participantes para poder establecer un vínculo con ellos.

\section{RESULTADOS}

Con respecto al objetivo de caracterizar los niveles de bienestar psicológico y autoeficacia generalizada antes y después de la aplicación del Programa Creciendo Fuertes, se presentan los resultados descriptivos de las variables "virtud del coraje", "autoeficacia generalizada" y "bienestar psicológico" PERMA con sus dimensiones: "emociones positivas", "flujo", "relaciones interpersonales", "sentido de vida", "logros" y "metas", en las medidas pre y post de la aplicación del Programa Creciendo Fuertes (véase tabla 1).

Tabla 1. Resultados pre y post Programa Creciendo Fuertes

\begin{tabular}{ccccccccc}
\hline \multirow{2}{*}{ Variables } & \multicolumn{4}{c}{ Medida PRE } & \multicolumn{3}{c}{ Medida POST } \\
\cline { 2 - 7 } & Min. & Max. & M & DE & Min. & Max. & M & DE \\
\hline Coraje & 74 & 120 & 95,45 & 10,66 & 56 & 112 & 95,79 & 13,95 \\
Emociones Positivas & 24 & 38 & 31,37 & 4,44 & 14 & 40 & 31,12 & 6,02 \\
Flujo & 13 & 23 & 19,54 & 2,76 & 11 & 25 & 20,50 & 3,37 \\
Relaciones Interpersonales & 24 & 39 & 31,79 & 3,85 & 11 & 40 & 31,75 & 6,75 \\
Sentido de vida & 19 & 43 & 31,45 & 5,61 & 17 & 45 & 34,33 & 6,28 \\
Logros y metas & 14 & 24 & 18,54 & 2,50 & 9 & 25 & 18,29 & 4,13 \\
Bienestar PeRMA & 105 & 167 & 132,70 & 15,85 & 66 & 169 & 136,00 & 22,93 \\
Autoeficacia Generalizada & 17 & 46 & 32,79 & 7,43 & 20 & 45 & 34,45 & 5,92 \\
\hline
\end{tabular}

Nota: N=24; Min.: puntuación minina; Max.: puntuación máxima; M: media; DE: desviación estándar.

Ahora bien, antes de avanzar en el tratamiento de los datos estadísticos se realizó la prueba de Shapiro-Wilk para contrastar la normalidad de la distribución de los datos. Se empleó esta prueba estadística debido al tamaño de las muestras $(n<30)$. Los resultados arrojaron valores de p mayores a 0,05, asumiendo la distribución normal de los datos, lo que permitió realizar análisis inferenciales paramétricos para todas las variables, con el fin de poder dar respuesta a los objetivos de investigación planteados. Por consiguiente, se empleó el coeficiente de correlación Pearson para dar respuesta al objetivo referido a analizar la relación de la virtud del coraje con el bienestar psicológico y la autoeficacia 
generalizada luego de realizado el Programa Creciendo Fuertes en los estudiantes participantes, y se obtuvieron correlaciones positivas, altas y significativas entre la virtud del coraje, autoeficacia y bienestar psicológico en cada una de sus dimensiones (véase tabla 2).

Tabla 2. Correlaciones entre las variables en la medida post

\begin{tabular}{lccccccc}
\hline \multicolumn{1}{c}{ Variables } & $\mathbf{1}$ & $\mathbf{2}$ & $\mathbf{3}$ & $\mathbf{4}$ & $\mathbf{5}$ & $\mathbf{6}$ & $\mathbf{7}$ \\
\hline 1. Virtud del coraje & 1,00 & & & & & & \\
2. Autoeficacia generalizada & $0,477^{*}$ & & & & & & \\
3. Bienestar PERMA & $0,668^{* *}$ & $0,638^{* *}$ & & & & & \\
4. Emociones positivas & 0,404 & $0,596^{* *}$ & $0,818^{* *}$ & & & & \\
5. Flujo & $0,578^{* *}$ & 0,340 & $0,693^{* *}$ & 0,354 & & & \\
6. Relaciones interpersonales & $0,576^{* *}$ & $0,572^{* *}$ & $0,848^{* *}$ & $0,601^{* *}$ & $0,553^{* *}$ & & \\
7. Sentido de vida & $0,615^{* *}$ & $0,516^{* *}$ & $0,890^{* *}$ & $0,668^{* *}$ & $0,530^{* *}$ & $0,654^{* *}$ & \\
8. Metas y logros & $0,611^{* *}$ & $0,572^{* *}$ & $0,815^{* *}$ & $0,587^{* *}$ & $0,615^{* *}$ & $0,688^{* *}$ & $0,617^{* *}$ \\
\hline
\end{tabular}

Nota: $* *=<0,001 *=<0,05$

Con respecto al objetivo de determinar el efecto del Programa Creciendo Fuertes sobre el bienestar psicológico y autoeficacia generalizada de adolescentes de secundaria, se realizaron análisis de prueba t para muestras dependientes. Se observan diferencias estadísticamente significativas en el promedio de las puntuaciones de bienestar psicológico antes y después de aplicar el programa $t(23)=-2.678, p<0,05$, así como también en autoeficacia generalizada: $t(23)=-2.857, p<0,05$, es decir que en cuanto a la efectividad del Programa Creciendo Fuertes basado en fortalezas del carácter hubo un incremento significativo en el nivel de bienestar psicológico y autoeficacia generalizada de estudiantes antes y después de participar en el programa.

\section{DISCUSIÓN Y CONCLUSIONES}

Con relación a caracterizar los niveles de bienestar psicológico antes y después del Programa Creciendo Fuertes se tienen incrementos que se verían expresados en que los estudiantes participantes tengan la posibilidad de experimentar mayores emociones positivas, vivenciar experiencias de flujo, mantener adecuadas relaciones sociales, encontrar significado a su vivencia subjetiva así como tener la posibilidad de identificar recursos para alcanzar metas y logros de acuerdo a la etapa evolutiva adolescente (Seligman, 2011), es decir que desde la perspectiva multidimensional estos estudiantes participantes del programa se autoperciben con adecuados niveles de bienestar psicológico. De igual manera, hubo aumento significativo en autoeficacia generalizada que se vería expresado en que los estudiantes participantes del programa se autoperciban como capaces de ejecutar tareas en situaciones concretas con propósitos establecidos, capacidad que les genera varias habilidades que deben ser organizadas eficazmente para alcanzar sus múltiples propósitos en la vida (Oliva et al., 2011). 
Asimismo, los resultados encontrados permitieron dar respuestas a las hipótesis planteadas referidas a que el Programa Creciendo Fuertes tiene efectos estadísticamente significativos en el bienestar psicológico y la autoeficacia generalizada de los adolescentes, incrementando el valor de sus medias luego de la aplicación del mismo, además se registran relaciones estadísticamente significativas entre la virtud de coraje, el bienestar psicológico y la autoeficacia generalizada en los estudiantes (véase tabla 2).

Con respecto a los niveles de relación entre la virtud del coraje y el bienestar psicológico, se comprobó que los resultados obtenidos son similares a los reportados en revisiones recientes presentados por Ovejero, Cardenal y Ortiz-Tallo (2016), en las cuales se plantea que las fortalezas del carácter presentan correlaciones con variables como el bienestar psicológico, la satisfacción vital y la resiliencia. En el caso específico de la correlación entre la virtud del coraje y el bienestar psicológico, en la revisión realizada por estos autores se presentan correlaciones de moderada a alta (desde 0,30 a 0,39). En el presente estudio se presentan correlaciones superiores entre estas variables, lo que mostró la relevancia de esta fortaleza en el desarrollo del potencial de los jóvenes. De modo que los hallazgos encontrados acerca de las relaciones significativas entre la virtud del coraje y bienestar psicológico son congruentes con los reportados por Park, Peterson y Seligman (2004); Peterson y Seligman (2004); Brdar y Kashdan (2010); Giménez (2010); Shimai, Otake, Park, Peterson y Seligman (2006); Polak y McCullough (2006); Peterson (2006); Sol Grinhauz (2012, 2015); Huta y Hawley (2008), Seligman, Steen, Park y Peterson (2005), Rojas (2010). Asimismo, otros estudios han puesto de manifiesto las relaciones significativas entre la autoeficacia y bienestar psicológico, a saber: Sansinenea et al. (2008); Galicia-Moyeda, Sánchez-Velasco y Robles-Ojeda (2013); Chiva (2017); Reigal, Videra y Gil (2014); Chavarría y Barra (2014); Cajiao, Morales, Garzón, Benavides y Acevedo (2013); González Cabanach, Valle, Freire y Ferradás (2012); Beas y Salanova (2004); García, Llorens, Salanova y Cifre (2006); Salanova, Martínez, Bresó, Llorens y Grau Gumbau (2005), y Bresó, Salanova, Martínez, Grau y Agut (2004), entre otros.

Del mismo modo, los hallazgos con respecto a la relación de la virtud del coraje y la autoeficacia generalizada se consideran pertinentes a la luz de la psicología evolutiva, debido a que ponen en relevancia las virtudes y fortalezas del carácter en el desarrollo de la autoeficacia generalizada en adolescentes. Esta afirmación se confirma en el estudio presentado, específicamente las fortalezas de la virtud del coraje. De igual forma, otros estudios han puesto de manifiesto las relaciones de las fortalezas del carácter con la autoeficacia, así Perandones González y Herrera Torres (2017); Ovejero, Cardenal y Ortiz-Tallo (2016); Perandones González, Herrera Torres y Lledó Carreres (2014), y Ruch, Weber, Park y Peterson (2014); Proctor, Linley y Maltby (2009). En ese sentido, Sánchez (2014, p. 10) expone, en una seria revisión sobre fortalezas del carácter en la adolescencia, que: "[...] cuantas más fortalezas posee un adolescente, más altas son las creencias de autoeficacia". En ese sentido, las fortalezas del carácter y los elementos del bienestar interactúan en el desarrollo y mantenimiento de la autoeficacia; entre ellos se mencionan el estado de ánimo positivo, el desarrollo de lazos sociales, compromiso, sentido de vida y satisfacción por los logros los cuales podrían servir de elementos protectores ante los posibles múltiples riesgos psicosociales a los que están expuestos los adolescentes. 
Ahora bien, el Programa Creciendo Fuertes, en concordancia con los planteamientos de Cuadra-Peralta et al. (2010), se inserta en la línea de los programas basados en Psicología Positiva que generan espacios o condiciones adecuadas para generar aumento en el bienestar psicológico, aunado a que se trabaja desde la voluntad para aprehender conceptos, técnicas y estrategias que ayudarían al desarrollo, crecimiento personal e incluso la sostenibilidad del bienestar a lo largo del tiempo mediante la puesta en prácticas de los aprendizajes, en este caso, el identificar, reconocer y ejecutar las fortalezas del carácter desde el contexto educacional como punto de inicio, pero buscando la extrapolación a otras dimensiones de la vida.

De igual manera, los resultados referidos de los efectos del Programa Creciendo Fuertes sobre el bienestar psicológico y autoeficacia generalizada en los estudiantes participantes, se pueden explicar también debido a que los programas de intervención en Psicología Positiva van orientados a que los sujetos realicen prácticas conscientes-voluntariosas de sus fortalezas del carácter (Bolier et al., 2013). Otros antecedentes refieren que el éxito de los programas de intervención en Psicología Positiva está basado en poner en práctica las fortalezas del carácter, así como la relación directa que tienen con el bienestar psicológico y otras variables de personalidad como optimismo, autoeficacia, emociones positivas y resiliencia (Sin y Lyubomirsky, 2009; Seligman et al., 2009; Cuadra-Peralta et al., 2010; Jiménez, Izal y Montorio, 2016).

Desde el punto de vista pragmático, estos resultados pueden tener implicaciones en los contextos educacionales con adolescentes en pro de mantener o mejorar su bienestar; es decir, con base en las correlaciones positivas significativas encontradas entre autoeficacia, bienestar psicológico y virtud del coraje, se pueden plantear vías de intervención educativas en distintas modalidades orientadas en estas variables que pueden ser: intervenciones cortas, cursos, talleres e incluso programas educativos orientados a la identificación, desarrollo y consolidación de rasgos en la adolescencia como el presentado en este estudio.

Desde el punto de vista el teórico-científico los resultados son congruentes con los postulados de la Psicología Positiva que explican que las fortalezas del carácter son rasgos de las virtudes humanas que las personas ponen en práctica en su vida cotidiana para cultivar el bienestar (Peterson y Seligman, 2004; Seligman, 2011). En efecto, se configuran como aporte al conocimiento desde estas teorías al contexto uruguayo. En ese sentido, García y Soler (2016) exponen que las fortalezas de la valentía, el autocontrol y la perseverancia pueden ayudar a los adolescentes en situaciones específicas para alcanzar objetivos, considerando los obstáculos que puedan originarse en el camino, y relacionándose a su vez con las dimensiones del bienestar psicológico, mientras que las fortalezas de la gratitud y la inteligencia social permiten a los adolescentes manejarse de manera adecuada en el mundo social relacionándose con la dimensión de relaciones del bienestar psicológico. 
Ahora bien, de acuerdo a los objetivos planteados se concluye que:

a. Hubo incremento en las características de los niveles de bienestar psicológico y autoeficacia generalizada antes y después de la aplicación del Programa Creciendo Fuertes.

b. Hubo un efecto estadísticamente significativo del Programa Creciendo Fuertes sobre el incremento en las medidas de bienestar psicológico y autoeficacia generalizada antes y después de la realización del mismo.

c. Existen correlaciones positivas, altas y significativas entre las variables "virtud del coraje", "bienestar psicológico" y "autoeficacia generalizada" en los estudiantes luego de la realización del programa.

De estas conclusiones, el principal aporte de este trabajo es determinar el efecto preliminar de un programa basado en fortalezas en el bienestar psicológico y autoeficacia generalizada en estudiantes adolescentes en contexto educacional uruguayo, con miras de ser implementado en distintas instituciones educativas con el firme propósito de generar factores de protección sobre el bienestar psicológico de los estudiantes ante factores y conductas de riesgo característicos de la etapa vital adolescente Como se mencionó anteriormente, los autores de este trabajo se ubican dentro del marco de la educación emocional, del carácter y para el bienestar, de modo que, esta experiencia de investigación basada en la promoción del bienestar psicológico en la secundaria, se configura como un pequeño grano de arena para ayudar a formar un movimiento científico educativo que busque en sus propósitos el desarrollo pleno de los estudiantes en la República Oriental del Uruguay.

Sin embargo, este estudio debe ser considerado preliminar debido a sus limitaciones en tamaño de la muestra y diseño de investigación. En efecto, la principal limitación fue la de no contar con un grupo de control; se informa que el diseño de investigación presentado en este estudio obedece a razones externas de los investigadores que impidieron el diseño experimental, pues la ejecución del programa se llevó a cabo en el medio natural de la institución educativa, haciendo imposible la asignación a azar de los participantes. Esto es difícil de lograr en los escenarios educativos, pues alcanzar la condición de grupo de control implicaría privar del Programa Creciendo Fuertes al menos a uno de los grupos de secundaria de la institución educativa, acarreando otros desafíos éticos en cuanto a la gestión educativa, como lo exponen León y Montero (2015). No obstante, estos resultados se perciben como alentadores, optimistas e incluso sugestivos para ejecutar el programa en otras instituciones educativas.

También se presentan otras futuras líneas de trabajo, por ejemplo, el estudiar el funcionamiento del Programa Creciendo Fuertes en un ciclo básico de secundaria completo, es decir, primer, segundo y tercer año, ya sea de manera transversal o inclusive longitudinal. De igual manera, estos resultados son motivadores para pensar en otros diseños de investigación más completos desde el punto de vista metodológico como la posible inclusión de grupos controles, diseñar una instancia bajo un diseño completamente experimental e incluso estudiar el programa con relación a otras variables dependientes en el marco de la educación emocional, del carácter y para el bienestar. 


\section{REFERENCIAS}

Alegre, A. (2013). Autoeficacia y procrastinación académica en estudiantes universitarios de Lima Metropolitana. Propósitos y Representaciones, 1(2), 57-82.

Arguís, R., Bolsas; A.P., Hernández, S. y Salvador, M. (2010). Programa Aulas Felices. Psicología Positiva aplicada a la educación. Recuperado de: http://catedu.es/ psicologiapositiva/

Bandura, A. (1997). Self-efficacy. The exercise of control. W.H. Friedman and co.

Bandura, A. (2011). A Social Cognitive perspective on Positive Psychology. Revista de Psicología Social, 26(1), 7-20. doi: https://doi.org/10.1174/021347411794078444

Bandura, A. (2012). On the Functional Properties of Perceived Self-Efficacy Revisited. Journal of Management, 38(1), 9-44. doi: 10.1177/0149206311410606

Beas, I.M. y Salanova, M. (2004). Autoeficacia y su relación con el bienestar psicológico y la formación con los ordenadores en usuarios de tecnología. En: M. Salanova, R. Grau, I.M. Martínez, E. Cifre, S. Llorens y M. García-Renedo (eds.). (2004). Nuevos horizontes en la investigación sobre autoeficacia. Castellón: Colección Psique, 8 (pp. 106-116).

Bernal, A.; González-Torres, M.C. y Naval, C. (2015). La educación del carácter. Perspectivas internacionales. Participación educativa. Revista del Consejo Escolar del Estado, 4 (6), 35-45.

Bisquerra Alzina, R. (2010). Educación Emocional y Bienestar. Barcelona: Edit. Praxis.

Bisquerra Alzina, R; Hernández Paniello, S. (2017). Psicología Positiva, educación emocional y el Programa Aulas Felices. Papeles del Psicólogo, 38(1), 58-65.

Bolier, L.; Haverman, M.; Westerhof, G.J.; Riper, H.; Smit, F. y Bohlmeijer, E. (2013). Positive psychology interventions: a meta-analysis of randomized controlled studies. BMC public health, 13(1), 119.

Brdar, I. y Kashdan, T. (2010). Character strengths and well-being in Croatia: An empirical investigation of structure and correlates. Journal of Research in Personality, 44(1),151-154

Bresó, E.; Salanova, M.; Martínez, I.M.; Grau, R. y Agut, S. (2004). Éxito académico y expectativas de éxito: el rol mediador de la autoeficacia académica. En: M. Salanova, R. Grau, I.M. Martínez, E. Cifre, S. Llorens y M. García-Renedo (eds.). (2004). Nuevos Horizontes en la investigación sobre Autoeficacia. Castellón: Colección Psique, 8, (pp. 237-243).

Cajiao, G.; Morales, D.; Garzón, G.; Benavides, L. y Acevedo, J. (2013). Revisión de algunos avances en la literatura sobre variables predictoras concernientes al "bienestar subjetivo". Cuadernos Hispanoamericanos de Psicología, 13(1), 57-62. 
García-Álvarez et al - Efectos del programa Creciendo Fuertes sobre el bienestar psicológico y autoeficacia

Chavarría, M. y Barra, E. (2014). Satisfacción vital en adolescentes: relación con la autoeficacia y el apoyo social percibido. Terapia psicológica, 32(1), 41-46.

Chiva, M. (2017). Bienestar psicológico, autoestima y autoeficacia general en el ámbito de la danza [Trabajo de grado inédito]. Madrid: Facultad de Ciencias Humanas y Sociales de la Universidad Pontificia Comillas.

Cobo, R. y García, D. (2017). La felicidad es educable: a propósito del bienestar en las escuelas. Revista Convocación, 33-34, VII, 38-47.

Contreras, F. y Esguerra, G. (2006). Psicología Positiva: una nueva perspectiva en psicología. Diversitas: perspectivas en psicología, 2(2), 311-319.

Csikszentmihalyi, M. (1997). Finding flow: The psychology of engagement with everyday life. Basic Books.

Cuadra-Peralta, A.; Veloso-Besio, C.; Moya, Y.; Reyes, L. y Vilca, J. (2010). Efecto de un programa de Psicología Positiva e inteligencia emocional sobre la satisfacción laboral y vital. Salud y Sociedad, 1(2), 101-112.

Díaz Barriga, Á. (2009). Pensar la didáctica. Buenos Aires: Amorrortu.

Fernández Ríos, L. (2008). Una revisión crítica de la Psicología Positiva: historia y concepto. Revista Colombiana de Psicología, 17, 161-176.

Galicia-Moyeda, I. y Sánchez-Velasco, A. y Robles-Ojeda, F. (2013). Autoeficacia en escolares adolescentes: su relación con la depresión, el rendimiento académico y las relaciones familiares. Anales de Psicología, 29(2), 491-500.

García, D. y Serrano, M. (2017). La inteligencia emocional: capacidad para el desarrollo humano en el marco de la orientación educativa. Revista Convocación, 30, VII, 56-67.

García, D. y Soler, M. (2016). Bienestar psicológico en la secundaria: estudio piloto con docentes y adolescentes. Revista Convocación, 29, VI, 34-44.

García, D. y Achard, L. (2017). Sobre Psicología Positiva en la educación: Programa Creciendo Fuertes. Revista Convocación, 32, VII, 54-65.

García, M.; Llorens, S.; Salanova, M. y Cifre, E. (2006). Antecedentes afectivos de la autoeficacia docente: un modelo de relaciones estructurales. Revista de Educación, $339,387-400$.

Giménez, M. (2010). La medida de las fortalezas psicológicas en adolescentes (VIAY): relación con clima familiar, psicopatología y bienestar psicológico [tesis doctoral inédita]. Madrid: Facultad de Psicología, Universidad Complutense.

González Cabanach, R.; Valle Arias, A.; Freire Rodríguez, C. y Ferradás Canedo, M. (2012). Relaciones entre la autoeficacia percibida y el bienestar psicológico en estudiantes universitarios. Revista Mexicana de Psicología, 29 (1), 40-48. 
Hernández, R.; Fernández, C. y Baptista, P. (2008). Metodología de la investigación científica. México: Mc Graw Hill.

Huta, V.; y Hawley, L. (2010). Psychological strengths and vulnerabilities: Are they two ends of the same continuum or do they have independent relationships with well-being and ill-being? Journal of Happiness Studies, 11, 71-93.

Jiménez, M.G.; Izal, M. y Montorio, I. (2016). Programa para la mejora del bienestar de las personas mayores. Estudio piloto basado en la Psicología Positiva. Suma psicológica, 23(1), 51-59.

León, O.G., y Montero, I. (2015). Metodología de investigación en psicología y educación. Las tradiciones cuantitativa y cualitativa. Madrid, España: Mc Graw Hill

Liccioni, E. y Soto, L. (2006). La educación emocional en niños, Estrategias para du desarrollo en la escuela. Valencia, Venezuela: Universidad de Carabobo

López, A. (2012). Resiliencia en niños y adolescentes venezolanos maltratados. En: Garassini, M. y Camilli, C. (eds.). La felicidad duradera. Estudios sobre el bienestar en la Psicología Positiva (pp. 87-117). Caracas: Editorial Alfa.

López, A. y Pérez, M. y D’Aubeterre, M. (2010). Resiliencia en niños y jóvenes: reconstruir esperanza. En: Garassini, M. y Camilli, C. (comp.). Psicología Positiva: Estudios en Venezuela (pp. 192-221). Caracas: Monfort.

Lyubomirsky, S. y Sheldon, K.M. (2005). Pursuing Happiness: The Architecture of Sustainable Change. Review of General Psychology, 9(2), 111-13. doi: 10.1037/10892680.9.2.111

McMahan, E.A. y Estes, D. (2011). Hedonic Versus Eudaimonic Conceptions of Well-being: Evidence of Differential Associations With Self-reported Well-being. Social Indicators Research, 103(1), 93-108. doi: 10.1007/s11205-010-9698-0

Montero, I. y León, O. (2002). Clasificación y descripción de las metodologías de investigación en psicología. Revista Internacional de Psicología Clínica y de la Salud, 2, 503-508.

Moreno, B.; Gálvez, M. (2010). La Psicología Positiva va a la escuela típica. Boletín Electrónico de Salud Escolar, 6(1), 210-220.

Muñoz de Morales, M. y Bisquerra, R. (2013). Diseño, aplicación y evaluación de un plan de educación emocional en Guipúzcoa: análisis cuantitativo. Revista EduPsykhé, 12(1), 3-21.

Oliva, A.; Antolín, L. ; Pertegal, M.A; Ríos, M. ; A. ; Parra, A.; Hernando, A. y Reina, M.C. (2011). Instrumentos para la evaluación de la salud mental y el desarrollo positivo adolescente y los activos que lo promueven. Sevilla: Consejería de Salud de la Junta de Andalucía. 
García-Álvarez et al - Efectos del programa Creciendo Fuertes sobre el bienestar psicológico y autoeficacia

Organización Mundial de la Salud. (2011). Prevenir el embarazo precoz y los resultados reproductivos adversos en adolescentes en los países en desarrollo: las evidencias. Suiza: OMS.

Ornelas Contreras, M.; Blanco Vega, H.; Peinado Pérez, J.E. y Blanco Ornelas, J.R. (2012). Autoeficacia percibida en conductas académicas en universitarios: un estudio respecto de alumnos de educación física. Revista mexicana de investigación educativa, $17,779-791$.

Ovejero, M.; Cardenal, V. y Ortiz-Tallo, M. (2016). Fortalezas humanas y bienestar biopsicosocial: revisión sistemática. Escritos de Psicología, 9(3), 4-14.

Palomera, R. (2017). Psicología Positiva en la escuela: un cambio con raíces profundas. Papeles del Psicólogo, 38(1), 66-71.

Papalia, D.; Feldman, R. y Martorell, G. (2012). Desarrollo humano. México D. F.: McGraw Hill.

Park, N.; Peterson, C. y Seligman, M. (2004). Strengths of character and well-being. Journal of Social y Clinical Psychology, 23, 603-619.

Peñacoba-Arribas, A. y Santa-Cruz Vera, D. (2016). El declive de la educación de la voluntad: problemática y tendencias educativas. Educación y Educadores, 19(3), 439457.

Perandones González, T. y Herrera Torres, L. (2017). Autoeficacia docente y fortalezas y virtudes personales en docentes de república dominicana. International Journal of Developmental and Educational Psychology, 3(1), 387-396.

Perandones González, T.; Herrera Torres, L. y Lledó Carreres, A. (2014). Fortalezas y virtudes personales del profesorado y su relación con la eficacia docente. International Journal of Developmental and Educational Psychology, 7(1), 141-150.

Peterson, C. (2006). A primer in positive psychology. New York: Oxford University Press

Peterson, C. y Seligman, P. (2004). Character strengths and virtues: a handbook and classification. Oxford: Oxford University Press y American Psychological Association.

Polak, E. y McCullough, M. (2006). Is gratitude an alternative to materialism? Journal of Happiness Studies, 7, 343-360.

Proctor, C.; Linley, P. y Maltby, J. (2009). Youth Life Satisfaction: A Review of the Literature. Journal of Happiness Studies, 10, 583-630.

Quiceno, D. y Vinaccia, S. (2013). Calidad de vida, factores salutogénicos e ideación suicida en adolescentes. Terapia Psicológica, 31(2), 263-271. Recuperado de: http:// www.scielo.cl/scielo.php?script $=$ sci_arttextypid $=$ S0718-48082013000200012ylng $=$ esy tlng=es. 10.4067/S0718-48082013000200012 
Reigal, R.; Videra, A. y Gil, J. (2014). Práctica física, autoeficacia general y satisfacción vital en la adolescencia. Revista Internacional de Medicina y Ciencias de la Actividad Física y del Deporte, 14(55), 561-576.

Rivers, I. y Cowie, H. (2006). Bullying and homophobia in UK schools: a perspective on factors affecting resilience and recovery. Journal of Gay and Lesbian Issues in Education, $3(4), 11-43$.

Rojas, R. (2010). Fortalezas del carácter, variables sociodemográficas y salud en trabajadores de sector público [tesis de maestría]. Caracas, Venezuela: Universidad Simón Bolívar, Maestría en Psicología.

Romero, A.; Cruz, S.; Gallardo, C. y Peñacoba, C. (2013). Cómo promocionar la salud y el bienestar en la comunidad universitaria. Universidad Rey Juan Carlos, universidad saludable. Revista Iberoamericana de Psicología y Salud, 4(2), 49-64.

Ruch, W.; Weber, M.; Park, N. y Peterson, C. (2014). Character strengths in children and adolescents: Reliability and initial validity of the german values in action inventory of strengths for youth (german VIA-youth). European Journal of Psychological Assessment, 30(1), 57-64.

Ruiz-Aranda, D.; Cabello, R.; Salguero, J.M.; Castillo, R.; Extremera, N. y FernándezBerrocal, P. (2010). Los adolescentes malagueños ante las drogas: la influencia de la inteligencia emocional. Madrid: TEA ediciones.

Salanova M.; Martínez, I.; Bresó, E.; Llorens, S. y Grau Gumbau, R. (2005). Bienestar psicológico en estudiantes universitarios: facilitadores y obstaculizadores del desempeño académico. Anales de Psicología, 21(1), 170-180.

Sánchez, C. (2014). Revisión teórica sobre el estudio de las fortalezas humanas en diferentes etapas evolutivas [trabajo de fin de grado]. Jaén, España: Universidad de Jaén.

Sansinenea, E.; Gil de Montes, L. ; Agirrezabal, A.; Larrañaga, M.; Ortiz, G. ; Valencia, J. y Fuster, M. (2008). Autoconcordancia y autoeficacia en los objetivos personales: ¿Cuál es su aportación al bienestar? Anales de Psicología, 24(1), 121-128.

Seligman, M. (2002). La auténtica felicidad. Barcelona: Grijalbo.

Seligman, M. (2011). Flourish: A Visionary New Understanding of Happiness and Wellbeing. Nueva York: Free Press.

Seligman, M.; Ernst, R.; Gillham, J.; Reivich, K. y Linkins, M. (2009). Positive education: positive psychology and classroom interventions. Oxford Review of Education, 35(3), 293-311. 
Seligman, M.; Steen, T.; Park, N. y Peterson, C. (2005). Positive psychology progress: Empirical validation of interventions. American Psychologist, 60, 410-421.

Shimai, S.; Otake, K.; Park, N.; Peterson, C. y Seligman, M.E.P. (2006). Convergence of character strengths in American and Japanese young adults. Journal of Happiness Studies, 7, 311-322.

Sin, N.L. y Lyubomirsky, S. (2009). Enhancing well being and alleviating depressive symptoms with positive psychology interventions: A practice friendly meta analysis. Journal of clinical psychology, 65(5), 467-487.

Sol Grinhauz, A. (2012). El análisis de las virtudes y fortalezas en niños: una breve revisión teórica. Psicodebate, 12, 39-61.

Sol Grinhauz, A. (2015). El estudio de las fortalezas del carácter en niños: Relaciones con el bienestar psicológico, la deseabilidad social y la personalidad. Psicodebate, 15(1), 43-68.

Soler, M. (2016). Virtud del coraje en relación al bienestar psicologico en adolescentes [tesis doctoral inédita]. Buenos Aires, Argentina: Facultad de Psicología, Universidad de Flores.

Toner, E.; Haslam, N.; Robinson, J. y Williams, P. (2012). Character Strengths and Wellbeing in Adolescents. Personality and Individual Differences, 52, 637-642.

Universidad Metropolitana y Fondo de Naciones Unidas para la Infancia. (2012). Adolescentes cuentan... Historias de líderes de su comunidad. Caracas: Editorial Ignaka. Vargas, L. y González-Torres, M. (2009) La revitalización de la educación del carácter en el ámbito psicoeducativo actual: aportaciones desde las ciencias de la prevención y la Psicología Positiva. Electronic Journal of Research in Educational Psychology, 19, 7(3), 1379-1418.

Yeager, J.M.; Fisher, S.W. y Shearon, D.N. (2011). Smart Strengths: A Parent-teachercoach Guide to Building Character, Resilience and Relationships in Youth. New York: Travis Pub. 